\title{
Identificación de propiedades presentes en jugo de Opuntia megacantha Salm-Dyck importantes para la producción de biopolímeros
}

\author{
Sandra Pascoe-Ortiz ${ }^{1}$, Ramón Rodríguez-Macías*2, Jorge Ramón Robledo-Ortiz ${ }^{3}$, \\ Eduardo Salcedo-Pérez ${ }^{2}$, Juan Francisco Zamora-Natera ${ }^{2}$, \\ Martín Rabelero-Velasco ${ }^{4}$ y J. Jesús Vargas-Radillo ${ }^{3}$ \\ ${ }^{1}$ Doctorado en Ciencias en Biosistemática, Ecología y Manejo de Recursos Naturales y Agrícolas. Centro \\ Universitario de Ciencias Biológicas y Agropecuarias, Universidad de Guadalajara, Nextipac, Zapopan \\ 44600, Jalisco, México. Jefatura de Investigación, Universidad del Valle de Atemajac, Prados Tepeyac 45050. \\ Zapopan, Jalisco, México. ${ }^{2}$ Departamento de Botánica y Zoología. Centro Universitario de Ciencias Biológicas \\ y Agropecuarias, Universidad de Guadalajara, Jalisco, México. ${ }^{3}$ Departamento de Madera, Celulosa y Papel. \\ Universidad de Guadalajara, Jalisco, México. ${ }^{4}$ Departamento de Ingeniería Química. Centro Universitario de \\ Ciencias Exactas e Ingenierías, Universidad de Guadalajara, Jalisco, México. *E:mail: ramonrod@cucba.udg.mx
}

\begin{abstract}
RESUMEN
Con el objetivo de identificar las propiedades químicas y los antioxidantes de interés en la industria de los biopolímeros se realizó la caracterización química del jugo de cuatro variantes de cladodios de Opuntia megacantha; las variantes presentan dos grados de madurez (tiernos y maduros) y dos manejos diferentes durante su desarrollo (silvestres y cultivados). El grado de madurez de los cladodios fue estadísticamente significativo ( $\mathrm{p} \leq 0.05)$, el jugo de los cladodios maduros presentó mayor cantidad de fibra cruda y material libre de nitrógeno, el jugo de cladodios tiernos contiene mayor cantidad de proteína, extracto etéreo y cenizas. No existió diferencia estadística significativa $(p=0.6917)$, en el contenido de los fenoles en las cuatro variantes se obtuvieron concentraciones entre 165.6 y $176.6 \mathrm{mg} \mathrm{GAE} / \mathrm{mL} ;(2.18$ y $3.61 \mathrm{mg}$ GAE$/ \mathrm{g}$ en peso seco). Los jugos de los cladodios maduros contienen mayor cantidad de los diferentes azúcares cuantificados $(\mathrm{p} \leq 0.05)$. Se prepararon películas de biopolímero utilizando jugo clarificado de cladodios silvestres tiernos para determinar su resistencia a la tensión con un resultado de 1.42 MPa, módulo de Young de 1.77 MPa y elongación a la rotura de $124.16 \%$; se concluye que los jugos caracterizados en este trabajo pueden ofrecer una alternativa viable a los polímeros derivados del petróleo.

Palabras Clave: Análisis proximal, metabolitos secundarios, tamizaje fitoquímico, monosacáridos, pruebas mecánicas.
\end{abstract}

\section{Identification of important properties present in juice of Opuntia megacantha} Salm-Dyck for the production of biopolymers

\begin{abstract}
In order to identify chemical and antioxidant properties of interest in the biopolymer industry, the chemical characterization of the juice of four variants of Opuntia megacantha cladodes was carried out; the variants present two degrees of maturity (tender and mature) and two different managements during their development (wild and cultivated). The degree of maturity of the cladodes was statistically significant $(\mathrm{p} \leq 0.05)$, the juice of mature cladodes presented a greater amount of crude fiber and nitrogen-free material, while the juice of tender cladodes contains a greater amount of protein, ethereal extract and ashes. There was no significant statistical difference $(p=0.6917)$, in the content of phenols in the four studied variants, with concentrations between 165.6 and $176.6 \mathrm{mg} \mathrm{GAE} / \mathrm{mL} ;(2.18$ and $3.61 \mathrm{mg} \mathrm{GAE} / \mathrm{g}$ in dry weight). Ripe cladodes juices contain more of the different quantified sugars $(\mathrm{p} \leq 0.05)$. Biopolymer films were prepared using clarified juice of tender wild cladodes, determining their tensile strength of $1.42 \mathrm{MPa}$, Young's modulus of 1.77 MPa and elongation at breakage of $124.16 \%$, it is considered that the juices characterized in this work can offer viable alternative to petroleum-derived polymers.

Key Words: Proximal analysis, secondary metabolites, phytochemical screening, monosaccharides mechanical tests.
\end{abstract}

Nota: Artículo recibido el 22 de julio del 2019 y aceptado el 06 de diciembre del 2019. 


\section{INTRODUCCIÓN}

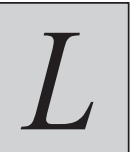

os nopales son originarios de América tropical y subtropical. Se conocen casi 300 especies del género Opuntia, de las cuales sólo 10 o 12 son utilizadas por el hombre en diferentes aplicaciones, entre ellas para productos alimenticios, fibras, mucílagos, pigmentos, vitaminas, colorantes, hidrocoloides, cosméticos, bioenergía, fertilizantes, pinturas y biopolímeros (Sáenz, 2006). De acuerdo con González, Bernal, Gutiérrez \& Santamaría, (2018) el nopal tiene usos y aplicaciones potenciales, las pencas de las podas de nopal tunero pueden utilizarse como forraje; si se procesan se obtiene fibra deshidratada, jugo con propiedades hipoglucemiantes y mucílago. El nopal ha sido motivo de diferentes estudios, "se puede considerar como un alimento funcional, es decir, mejora la salud de quienes lo consumen" (Valencia, Brambila \& Mora, 2010), de acuerdo con Torres, Morales, Ballinas \& Nevárez, 2015), se utiliza en la industria farmacéutica por su efecto hipoglucemiante y por sus propiedades nutracéuticas, como aditivo en la industria alimentaria y en la nutrición animal; es también utilizado con fines medicinales (Knishinsky, 2004).

Los contenidos nutrimentales del nopal varían de acuerdo a la especie y al grado de madurez de la misma, aunque mucho más a la madurez; los nopales más jóvenes presentan mejores características nutrimentales, independientemente de la especie (Ramírez, Reyes, Pinos \& Aguirre, 2007). Se sabe que el manejo humano de los procesos de cultivo genera cambios genéticos, morfológicos y en las características químicas de las plantas (Casas, Caballero, Valiente, Soriano \& Dávila, 1999). Las diferentes especies del género Opuntia tienen gran cantidad de hidrocoloides como las gomas, mucílagos y polisacáridos estructurales en forma de fibras; todos son importantes para las funciones fisiológicas y de protección de la planta (Reid, 1985; Nobel, Cavelier \& Andrade, 1992; Loveys, Stoll \& Davies, 2004).

Algunos investigadores han propuesto que la especie $O$. megacantha Salm-Dick, 1834, cuyo nombre vulgar es Nopal de Castilla o fafayuco es la forma silvestre de O. ficus-indica; es una planta arbórea cuyos artículos son de color verde claro a glauco, aplanados y miden entre 30 y $40 \mathrm{~cm}$ de largo y de 22 a $27 \mathrm{~cm}$ de ancho; con espinas subuladas, aplanadas de 1 a $3 \mathrm{~cm}$ de largo y escasas; con aréolas pequeñas distantes entre sí de 3.3 a $5 \mathrm{~cm}$, rodeadas de un margen de fieltro blanco grisáceo y en el centro fieltro castaño claro (González, Riojas \& Arreola, 2001), se le considera una especie semidomesticada (García et al., 2015).

En 2014, en México se cultivaron 84,558.1 ha con nopal, que produjeron 140,045.38 ton. de nopal forrajero, 824,602.36 ton. de nopal verdura, 568,404.9 ton. de tuna y continúa incrementándose la producción (SAGARPA, 2015). La alcaldía de Milpa Alta es la principal productora de nopal verdura, produciendo alrededor de 350, 000 ton. al año con un rendimiento de 90 ton/ha (SAGARPA, 2016). De acuerdo con Torres (2010), Brasil es el país con mayor superficie de nopal cultivado en el mudo, contando con más de 600, 000 ha, el nopal producido en este país es prácticamente forrajero.

Actualmente en diferentes partes del mundo, se llevan a cabo investigaciones para producir a partir del nopal, plásticos de origen natural que puedan usarse de diversas maneras, se ha tratado de igualar las propiedades físico-mecánicas de los plásticos convencionales esperando que en corto tiempo se logre su incorporación a los mercados y su utilización sea una opción rentable (Carballo, 2009). Los biopolímeros o (bioplásticos) son materiales con propiedades similares a los plásticos derivados del petróleo, pero que se producen a partir de fuentes de carbono renovables (azúcares, ácidos, lípidos, etc.) y son generalmente biodegradables. Se clasifican de acuerdo a su origen y al proceso de síntesis por el cual son obtenidos (Tharanathan, 2003; Valero, Ortegón \& Uscategui, 2013). Se ha encontrado que los polisacáridos propios de algunas plantas, pueden ser utilizados como materia prima para la elaboración de plásticos naturales; dentro de los polisacáridos que comúnmente se han estudiado con este fin, se encuentra el almidón que está presente en muchos vegetales, entre ellos el plátano (Zamudio, Bello, Vargas, Hernández \& Romero, 2007) y la yuca (Ruiz, 2006). Otro polisacárido que ha sido estudiado con este fin, es el mucílago de diferentes plantas entre las que destacan el nopal (Espino et al., 2010) y la sábila (Restrepo \& Aristizábal, 2010), ambas utilizadas principalmente en la producción de películas plásticas con el fin de recubrir alimentos.

Se ha reportado actividad antioxidante en los compuestos fenólicos presentes tanto en los cladodios como en los frutos de diferentes especies del género Opuntia (Ndhlala et al., 2007; Figueroa et al., 2010; Moussa-Ayoub, El-Samahy, Rohn \& Kroh, 2011; Gallegos, Scheinvar, Núñez \& Mondragón, 2012; Yeddes, Chérif, Guyot, Sotin \& Ayadi, 2013).

El presente trabajo, tiene por objetivo conocer el efecto de la madurez y tipo de cultivo sobre la composición de los macrocomponentes y antioxidantes del jugo de O. megacantha y evaluar la factibilidad para obtener biopolímeros a partir de él.

\section{Materiales Y MÉTOdos Material vegetal}

En este estudio se utilizaron cuatro variantes de nopal de la especie $O$. megacantha, las cuales se colectaron en el rancho llamado Las Papas de Arriba, del municipio de Ojuelos de Jalisco, Jalisco, México (2143'51.2”N 10140'16.1”WO), entre los meses de mayo y julio de 2016 (en esta zona las condiciones medioambientales registradas durante el año son las siguientes: Temperatura $=16.5{ }^{\circ} \mathrm{C}$, Precipitación 
pluvial $=44 \mathrm{~mm}$ en promedio). Para el estudio, se colectó nopal silvestre tierno (ST), silvestre maduro (SM), cultivado tierno (CT) y cultivado maduro (CM). El término "tierno" se aplica a cladodios de 0 a 3 meses de edad y el término "maduro" se aplica para cladodios de 12 a 15 meses de edad. El método de extracción de jugo utilizado consistió en lavar el nopal, retirar las espinas, molerlo en un extractor de jugos y dejarlo decantar por una semana en refrigeración a $4{ }^{\circ} \mathrm{C}$. El jugo clarificado se conservó congelado en recipientes de plástico hasta su utilización.

\section{Análisis químico del jugo clarificado}

Se evaluó la humedad y materia volátil (AOAC 930.36), proteína (AOAC 954.04), extracto etéreo (AOAC 954.02), cenizas (AOAC 942.05), fibra cruda (AOAC 962.09), extracto libre de nitrógeno (por diferencia) y materia seca (por diferencia), todas ellas fueron realizadas por duplicado.

\section{Análisis fitoquímico}

Para la determinación de los metabolitos secundarios (flavonoides, fenoles, cumarinas, terpenoides, alcaloides, carbohidratos, entre otros), se llevó a cabo un tamizaje fitoquímico empleando técnicas tradicionales (Harborne, 1998). La presencia de flavonoides se evaluó mediante la prueba de Shinoda, flavonas y flavonoles por medio de la prueba de Salkowski, esteroles y terpenoides por el método de Libermann-Burchard, alcaloides por los métodos de Wagner y Dragendorff, fenoles por la prueba de nitrato sódico, taninos con los métodos de gelatina y cloruro férrico, saponinas con la técnica de espuma, cumarinas por medio de los métodos de Ehrlich e hidróxido de sodio, quinonas con las técnicas de ácido sulfúrico e hidróxido de amonio, carbohidratos por Molisch, azúcares reductores con Fehling, pentosas y ácidos urónicos por la técnica de vial y la hidrólisis de la sacarosa con ácido clorhídrico. Las pruebas se llevaron a cabo por triplicado.

\section{Determinación de fenoles}

La cuantificación de fenoles totales se llevó a cabo mediante la técnica espectrofotométrica de Folin-Ciocalteu utilizando el ácido gálico como referencia, de acuerdo a Waterman \& Mole (1994). Se tomaron $0.5 \mathrm{~mL}$ de jugo clarificado y se mezcló con $0.75 \mathrm{~mL}$ de reactivo de Folin-Ciocalteu $1 \mathrm{~N}$ previamente preparado con una dilución del reactivo de Folin-Ciocalteu 2 N (Sigma-Aldrich). Se dejó reposar a temperatura ambiente 5 minutos y en seguida se agregaron $0.75 \mathrm{~mL}$ de solución de carbonato de sodio (Baker) al 20\%; se trató con ultrasonido 5 minutos y se dejó reposar 90 minutos a temperatura ambiente. Posteriormente se realizó la dilución en proporción 1:50 con agua destilada y se midió la absorbancia a $760 \mathrm{~nm}$ en un espectrofotómetro (VELAB modelo VE-5100UV). Los resultados se expresan en $\mathrm{mg}$ de ácido gálico equivalente por $\mathrm{mL}$ (mg GAE $/ \mathrm{mL}$ ) y $\mathrm{mg}$ de ácido gálico equivalente por $\mathrm{g}$ (mg GAE/g), el análisis se realizó por duplicado.

\section{Determinación de azúcares}

El jugo clarificado y filtrado de $O$. megacantha fue analizado por medio de cromatografía líquida de alta resolución, en un cromatógrafo SHIMADZU modelo SCL-10A con una columna Aminex HPX-87P, utilizando un detector de índice de refracción (RID). Se utilizó como fase móvil agua desionizada a un flujo de $0.6 \mathrm{~mL} / \mathrm{min}$. Los estándares utilizados para la identificación de carbohidratos fueron D-glucosa, D-galactosa, L-arabinosa, D-xilosa, L-ramnosa monohidratada, ácido D-galacturónico y ácido glucurónico, todos ellos de la marca Sigma-Aldrich. Se realizó una curva de calibración de cada monosacárido, para la identificación y cuantificación de los presentes en la muestra problema, las corridas se realizaron por duplicado.

\section{Muestras y pruebas mecánicas de un biopolímero}

Las muestras a partir de un biopolímero se elaboraron mezclando el jugo decantado de cladodios silvestres tiernos con proteína animal, glicerol y cera natural, manteniendo una proporción de 3:1:0.5:0.5 sobre una parrilla con agitación a $60{ }^{\circ} \mathrm{C}$. Se generaron películas por medio de la técnica de gel casting de acuerdo a las especificaciones de la norma ASTM D882 y se evaluaron las pruebas mecánicas de resistencia a la tensión, módulo de Young y Elongación a la rotura mediante un ensayo de tracción realizado en una máquina universal modelo 4411, todos los ensayos fueron realizados por duplicado y se reporta el promedio de los resultados en cada prueba.

\section{Estadística}

Para la comparación de las características químicas de los jugos se utilizó un diseño completamente al azar, considerándose como variables experimentales el grado de madurez y forma de cultivo del nopal y como variables dependientes los diferentes análisis hechos al jugo.

Los resultados se analizaron estadísticamente utilizando la prueba de ANOVA y la comparación de medias por Tukey con un nivel de confiabilidad de 95\%, en el software Statgraphics Centurion 15.

\section{RESULTADOS Y DISCUSIÓN \\ Rendimiento del jugo}

De los cladodios de nopal silvestre tierno (ST), se obtuvo el mayor rendimiento de jugo con un $62 \%$ en promedio, mientras que en los cladodios de nopal silvestre maduro (SM) y cultivado tierno (CT), el rendimiento fue el más bajo con un promedio de $44 \%$, intermedios se encuentran los cladodios de nopal cultivado maduro (CM), con un rendimiento del $54 \%$.

\section{Análisis químico}

Los resultados se presentan en la Tabla I. Se obtuvo diferencia estadística significativa en todos los parámetros determinados $(\mathrm{p} \leq 0.05)$. Los contenidos de proteína y extracto etéreo de 


\begin{tabular}{|l|c|c|c|c|}
\hline \multicolumn{1}{|c|}{ Parámetro } & ST & SM & CT & CM \\
\hline Humedad (\%) & $2.39^{\mathrm{a}}$ & $11.11^{\mathrm{b}}$ & $3.32^{\mathrm{a}}$ & $11.92^{\mathrm{b}}$ \\
\hline Proteína (\%N X 6.25) & $14.74^{\mathrm{b}}$ & $3.88^{\mathrm{a}}$ & $11.59^{\mathrm{b}}$ & $4.67^{\mathrm{a}}$ \\
\hline Extracto etéreo (\%) & $2.65^{\mathrm{d}}$ & $0.25^{\mathrm{a}}$ & $1.62^{\mathrm{c}}$ & $0.93^{\mathrm{b}}$ \\
\hline Cenizas (\%) & $23.56^{\mathrm{c}}$ & $17.46^{\mathrm{b}}$ & $26.88^{\mathrm{d}}$ & $14.82^{\mathrm{a}}$ \\
\hline Fibra cruda (\%) & $1.64^{\mathrm{a}}$ & $4.2^{\mathrm{b}}$ & $1.93^{\mathrm{a}}$ & $4.34^{\mathrm{b}}$ \\
\hline Extracto libre de nitrógeno (\%) & $55.02^{\mathrm{a}}$ & $63.1^{\mathrm{b}}$ & $54.66^{\mathrm{a}}$ & $64.04^{\mathrm{b}}$ \\
\hline Materia seca (\%) & $97.61^{\mathrm{b}}$ & $88.89^{\mathrm{a}}$ & $96.68^{\mathrm{b}}$ & $88.08^{\mathrm{a}}$ \\
\hline
\end{tabular}

$\mathrm{ST}=$ Silvestre Tierno; $\mathrm{SM}=$ Silvestre Maduro; $\mathrm{CT}=$ Cultivado Tierno; $\mathrm{CM}=$ Cultivado Maduro.

Tabla I. Análisis químico de jugo de nopal de $O$. megacantha. Letras diferentes en los datos de las columnas representan diferencia estadística significativa.

los jugos de cladodios tiernos ST y CT se presentan en mayor cantidad, en comparación con los presentes en los jugos de cladodios maduros SM y CM. De las muestras analizadas, la muestra ST es la que presenta mayor porcentaje en relación al contenido de proteínas y lípidos. Este resultado referente al mayor contenido de estos dos parámetros en cuanto a lípidos y proteínas coincide con el encontrado por otros investigadores; Contreras et al. (2012), que establecen que el contenido de extracto etéreo (lípidos), disminuye con la edad; de igual manera, se ha observado que en algunas especies de nopal la concentración de proteína disminuye con la edad del cladodio (Rodríguez et al., 2007; Sáenz, 1997), en este caso se encontró el mismo comportamiento al analizar el jugo de $O$. megacantha. Por otra parte, en relación a la fibra cruda, las variantes maduras tienen mayor contenido, lo que concuerda con los resultados de Pimienta (1990), en los que la fibra cruda se incrementa al aumentar la edad del cladodio. De acuerdo con Mondragón \& Pérez (2003), en los forrajes entre ellos el nopal, el contenido de nutrientes valiosos, en este caso la proteína disminuye con la edad debido al aumento de la cantidad de fibra. Así mismo, el extracto libre de nitrógeno (carbohidratos), presenta la misma tendencia que la fibra cruda, siendo los jugos de cladodios maduros los que presentan mayor contenido, lo cual es coincidente con lo reportado por Ribeiro et al. (2010), quienes encontraron que los cladodios maduros contienen mayor cantidad de azúcares. Finalmente, para el caso del contenido de cenizas el jugo $\mathrm{CT}$ presenta mayor cantidad, siendo el jugo $\mathrm{CM}$ el que tiene menor porcentaje Guzmán \& Chávez (2007) reportan que la especie Opuntia ficus-indica presenta calcio, potasio, sodio y hierro siendo los brotes maduros de $O$. ficus-indica los que presentan un incremento en las cenizas respecto a los brotes tiernos; para la especie $O$. megacantha del presente estudio resulta lo contrario.

De acuerdo con Fabra, Talens \& Chiralt (2010), las propiedades mecánicas como son: resistencia a la tensión, módulo de elasticidad y alargamiento de las películas plásticas, se relacionan con la composición química de las mismas. En un estudio que se hizo, las películas adicionadas con lípidos producen resistencia a la tensión y módulo de elasticidad menores, contrario a lo que sucedió con el alargamiento. En las películas plásticas existen interacciones moleculares entre lípidos y proteínas que favorecen el aumento de la resistencia y flexibilidad.

\section{Análisis fitoquímico}

En la tabla II están los resultados de las diferentes pruebas, se utilizó el sistema de cruces para denotar la presencia o ausencia del metabolito en la muestra. Se observa la presencia moderada de cumarinas en los jugos provenientes de cladodios maduros y son escasas en los jugos de cladodios tiernos, además se observa la presencia escasa de fenoles en los cuatro tipos de jugo, mientras que se presentaron carbohidratos abundantes en todos ellos, Torres et al. (2015), aseguran que el nopal contiene diferentes carbohidratos. En cuanto a los azúcares reductores, pentosas y ácidos urónicos, se presentan en forma abundante en el jugo SM, en forma moderada en el jugo CM y escasa en los jugos de los cladodios tiernos. Para la prueba de hidrólisis de sacarosa se observa que los jugos obtenidos de cladodios tiernos presentan abundancia de sacarosa, mientras que los jugos de cladodios maduros tienen una cantidad moderada de la misma. No se detectó presencia de flavonoides en ninguno de los cuatro jugos analizados, Fernández, Almeda, Obón \& Castellar (2010), mencionan que el contenido de estos compuestos puede depender de la especie o la forma de cultivo. En el trabajo de López \& Peña (2016) se reportan contenidos de fenoles, flavonoides y terpenoides en diferentes especies de Opuntia, entre ellas $O$. megacantha, sin embargo, en el presente trabajo sólo se detectó la presencia de fenoles.

\section{Determinación de fenoles}

El contenido de fenoles totales en las muestras analizadas no presentó diferencia estadística significativa, se encontró que varía entre 2.18 y $3.61 \mathrm{mg} \mathrm{GAE} / \mathrm{g}$; (Tabla III). Existen 


\begin{tabular}{|c|c|c|c|c|}
\hline Metabolito Secundario & ST & SM & CT & $\mathbf{C M}$ \\
\hline Cumarinas (Ehrlich) & + & ++ & + & ++ \\
\hline Cumarinas $(\mathrm{KOH})$ & + & + & + & + \\
\hline Quinonas $\left(\mathrm{H}_{2} \mathrm{SO}_{4}\right)$ & - & - & - & - \\
\hline Quinonas $\left(\mathrm{NH}_{4} \mathrm{OH}\right)$ & - & - & - & - \\
\hline Carbohidratos & +++ & +++ & +++ & +++ \\
\hline Azúcares reductores & + & +++ & + & ++ \\
\hline Pentosas y ácidos urónicos & + & +++ & + & ++ \\
\hline Hidrólisis sacarosa & +++ & ++ & +++ & ++ \\
\hline Flavonoides & - & - & - & - \\
\hline Flavonas y flavonoles & - & - & - & - \\
\hline Esteroles y triterpenos & - & - & - & - \\
\hline Alcaloides (Wagner) & - & - & - & - \\
\hline Alcaloides (Dragendorff) & - & - & - & - \\
\hline Fenoles & + & + & + & + \\
\hline Taninos (Gelatina) & - & - & - & - \\
\hline Taninos $\left(\mathrm{FeCl}_{3}\right)$ & - & - & - & - \\
\hline Saponinas & - & - & - & - \\
\hline
\end{tabular}

$(+++)$ Abundante, $(++)$ Moderado, $(+)$ Escaso, $(-)$ Negativo, $\mathrm{ST}=$ Silvestre Tierno; $\mathrm{SM}=$ Silvestre Maduro; $\mathrm{CT}=\mathrm{Cultivado}$ Tierno; $\mathrm{CM}=\mathrm{Cultivado}$ Maduro.

Tabla II. Resultados del análisis fitoquímico de jugo de nopal de $O$. megacantha.

\begin{tabular}{|c|c|c|}
\hline Jugo & $\begin{array}{c}\text { Fenoles Totales } \\
\text { (mg GAE/mL) }\end{array}$ & $\begin{array}{c}\text { Fenoles Totales } \\
\text { (mg GAE/g) }\end{array}$ \\
\hline ST & $174.9 \pm 5.0$ & $3.61 \pm 0.10$ \\
\hline SM & $165.6+10.2$ & $2.18 \pm 0.13$ \\
\hline CT & $176.6 \pm 14.2$ & $2.84 \pm 0.23$ \\
\hline CM & $174.1+15.5$ & $2.73 \pm 0.24$ \\
\hline
\end{tabular}

$\mathrm{ST}=$ Silvestre Tierno; $\mathrm{SM}=$ Silvestre Maduro $\mathrm{CT}=$ Cultivado Tierno; $\mathrm{CM}=$ Cultivado Maduro.

Tabla III. Resultados de la determinación de fenoles totales en jugo de $O$. megacantha.

algunos trabajos realizados con cladodios, en el de Flores, Vergara \& Guerrero (2011), se reportan fenoles totales entre 33.55 y $45.44 \mathrm{mg}$ GAE/g para cladodios frescos de Opuntia cochenillifera, el contenido difiere por la especie y porque el jugo sólo contiene una parte de los fenoles totales del cladodio. En otro trabajo (Apodaca, Martínez, Robles \& Rodríguez, 2016), encontraron diferencias significativas en el contenido de fenoles totales de acuerdo a la variedad y al grado de desarrollo de los cladodios del nopal verdura. Ellos sugieren que las diferencias encontradas entre las variedades son intrínsecas a la variedad en contraste con otros estudios (Guevara et al., 2010), que sugieren que las diferencias se deben al clima; en el presente trabajo al estudiar la misma variedad y colectarse en la misma plantación no existieron diferencias estadísticas en la cantidad de fenoles totales. Existen reportes de la cantidad de fenoles en el jugo de frutos de diferentes especies de Opuntia (Coria, Ochoa \& Nazareno, 2011), encontraron un contenido total entre 0.54 y $1.2 \mathrm{mg}$ GAE/g en jugo de tunas púrpura de Opuntia spp, púrpura oscuro de $O$. ficus-indica y naranjas de $O$. megacantha, siendo estas últimas las que presentaron los niveles más altos; Repo \& Encina (2008), encontraron en tunas rojas de Perú $52 \mathrm{mg} \mathrm{GAE} / 100 \mathrm{~g}$ cuyo contenido en ambos estudios fue menor a los encontrados en el jugo de $O$. megacantha de este trabajo, por otro lado (Chávez, Gutiérrez \& Serna, 2009; Sumaya et al., 2011), reportan cantidades de fenoles totales en el jugo de tunas amarillas, verdes y rojas semejantes a las del jugo de $O$. megacantha de este trabajo; Ndhlala et al. (2007) y Figueroa et al. (2010), encontraron mayor cantidad de fenoles totales, entre 334 y 2,262 mg GAE/g; y 141.44 y $420.66 \mathrm{mg} / \mathrm{L}$ respectivamente en tunas de Opuntia spp. comparada con la cantidad encontrada en $O$. megacantha de este estudio, pero menor en el jugo de tunas blancas (entre 14.24 y $22.78 \mathrm{mg} / \mathrm{L}$ ); algo similar a los resultados de los trabajos de Abdel-Hameed, Nagaty, Salman \& Bazaid (2014) y Jiménez, López, Hernández, Gutiérrez \& Welti (2015), en los cuales se reportan cantidades mayores de fenoles totales en los jugos de 
pulpa y de cáscaras de tunas rojas, verdes y amarillas entre 667.82 y $1152.97 \mathrm{mg}$ GAE/100 mL; y entre 1.89 y 12.75 g GAE/kg respectivamente, comparadas con las cantidades encontradas en este trabajo en el jugo de cladodios de $O$. megacantha; lo mismo sucede con la cantidad de fenoles totales para pulpa y cáscara de xoconostles (Osorio, Ortiz, Álvarez, Dorantes \& Giusti, 2011), de 15.01 y 19.9 mg $\mathrm{GAE} / \mathrm{g}$ respectivamente, que es mayor a la encontrada en esta investigación para $O$. megacantha. Estos reportes señalan que los compuestos fenólicos actúan como defensa de la planta contra la luz ultravioleta y como prevención del eventual ataque de patógenos.

La actividad antioxidante está correlacionada a la cantidad de fenoles totales (Pan et al., 2008), esto ha sido motivo de estudio en la producción de películas comestibles de almidón (Cenobio, Pimentel, Vargas, Güemes \& Campos, 2015), quienes mencionan que los compuestos fenólicos se utilizan en envases de alimentos debido a que disminuyen la oxidación y la producción de microorganismos; y en la producción de películas de quitosano (Wang, Dong, Men, Tong \& Zhou, 2008), ellos afirman que los compuestos fenólicos quedan inmersos en la matriz del polímero de acuerdo a la cantidad de extracto adicionado y disminuyen con el paso del tiempo. De acuerdo con Ciannamea, Stefani \& Ruseckaite (2016), algunos de los compuestos fenólicos pueden reaccionar con proteínas y favorecer la formación de películas comestibles, ya que actúan como plastificantes, además de que favorecen las propiedades antioxidantes de los biopolímeros obtenidos; Arcan \&Yemenicioglu (2011), afirman que los compuestos fenólicos pueden aumentar la flexibilidad de las películas por el efecto plastificante. De acuerdo con Nie, Gong, Wang \& Meng (2015), si se incorporan fenoles a las películas proteicas se puede aumentar la flexibilidad y reducir la permeabilidad al vapor de agua. Los compuestos fenólicos pueden enlazarse con polisacáridos y proteínas, ya que poseen anillos aromáticos hidrofóbicos y grupos hidroxilohidrofílicos que favorecen la formación de puentes de hidrógeno (Saura, 2010).

\section{Determinación de azúcares}

Los resultados se presentan en la Tabla IV, el análisis permite observar que la muestra CT contiene el mayor número de azúcares, mientras que la muestra SM contiene la menor cantidad de monosacáridos: ácido glucurónico, D-glucosa y D-galactosa, aunque en mayor concentración. Los jugos SM y CM presentan las más altas concentraciones de los monosacáridos que contienen. Sólo en el jugo de $O$. megacantha cultivado tierno (CT), fue posible la detección de D-glucosa, para los demás no fue detectable. López, Peña, Rodríguez \& Reyes (2016), reportan diferencias en los contenidos de azúcares presentes en las especies de nopal silvestre y cultivado, lo que coincide con los resultados de esta investigación donde se encontraron diferencias estadísticas en los contenidos de azúcares de las diferentes variantes estudiadas, no sólo por el manejo sino también en cuanto al grado de madurez. Paulsen \& Lund (1979) al igual que Trachtenberg \& Mayer (1981), reportan que el mucílago de $O$. ficus-indica contiene L-arabinosa, D-galactosa, ácido D-galacturónico, L- ramnosa y D-xilosa, siendo la D-galactosa la que está presente en mayor cantidad. En este trabajo D-galactosa estuvo presente en los cuatro tipos de jugos estudiados. Así mismo, el jugo de $O$. megacantha cultivado maduro (CM) fue el único en el cual se ha encontrado cierta concentración de L-ramnosa, mientras que en el resto de los jugos no fue detectable este monosacárido, esto es relevante, ya que en otros estudios (Ribeiro et al., 2010), en ningún tipo de jugo fue detectable el azúcar D-xilosa. De acuerdo con McGarvie \& Parolis (1979), la especie $O$. megacantha contiene L-arabinosa, D-galactosa, L-ramnosa, ácido glucurónico y trazas de D-xilosa coincidiendo con los resultados del presente trabajo.

Los polisacáridos tienen diferentes aplicaciones en la industria alimenticia, entre ellas se encuentra: la elaboración de películas comestibles para recubrimientos, las gomas guar, gellan y xantana que han sido estudiadas para estas aplicaciones (Domínguez \& Jiménez, 2012), estas gomas incorporan algunos monosacáridos presentes en el jugo de $O$. megacantha

\begin{tabular}{|l|c|c|c|c|}
\hline \multicolumn{1}{|c|}{ Azúcar, $\mathbf{~ m g / L ~}$} & ST & SM & CT & CM \\
\hline Ácido glucurónico & $756.6^{\mathrm{a}}$ & $4092.5^{\mathrm{d}}$ & $839.6^{\mathrm{b}}$ & $2327.0^{\mathrm{c}}$ \\
\hline Ácido D-galacturónico & $344.3^{\mathrm{b}}$ & $\mathrm{ND}$ & $236.3^{\mathrm{a}}$ & $407.2^{\mathrm{c}}$ \\
\hline D-glucosa & $\mathrm{ND}$ & $3311.5^{\mathrm{a}}$ & $\mathrm{ND}$ & $\mathrm{ND}$ \\
\hline D-galactosa & $252.9^{\mathrm{b}}$ & $2996.7^{\mathrm{d}}$ & $46.7^{\mathrm{a}}$ & $347.9^{\mathrm{c}}$ \\
\hline L-arabinosa & $91.0^{\mathrm{a}}$ & $\mathrm{ND}$ & $93.0^{\mathrm{a}}$ & $215.4^{\mathrm{b}}$ \\
\hline L-ramnosa & $\mathrm{ND}$ & $\mathrm{ND}$ & $52.1^{\mathrm{a}}$ & $\mathrm{ND}$ \\
\hline D-xilosa & $\mathrm{ND}$ & $\mathrm{ND}$ & $\mathrm{ND}$ & $\mathrm{ND}$ \\
\hline
\end{tabular}

$\mathrm{ST}=$ Silvestre Tierno; $\mathrm{SM}=$ Silvestre Maduro $\mathrm{CT}=$ Cultivado Tierno $; \mathrm{CM}=$ Cultivado Maduro, ND: No Detectable .

Tabla IV. Concentración de azúcares en jugo de $O$. megacantha. Los resultados en la misma fila con diferentes letras indican diferencia estadística significativa. 
como la D-galactosa, el ácido glucurónico, L-ramnosa y D-glucosa. En el trabajo de Saberi, Chockchaisawasdee, Golding, Scarlett \& Stathpoulos (2017), se encontró que D-galactosa tiene un efecto plastificante importante en películas comestibles de almidón y goma guar; en general los monosacáridos mostraron características comparables a las de polioles comúnmente utilizados como plastificantes en cuanto a las propiedades mecánicas, de barrera y ópticas.

\section{Pruebas mecánicas del biopolímero}

Los resultados de las pruebas mecánicas en películas formuladas con jugo clarificado de cladodios silvestres tiernos de $O$. megacantha, se desprenden del ensayo de tracción que se muestra en la Gráfica 1. Se tuvo una resistencia a la tensión de $1.42 \pm 0.17 \mathrm{MPa}$ semejante a las de las películas de albúmina de huevo (Gennadios, Brandenburg, Weller \& Testin, 1993), del gluten de trigo (Gennadios et al., 1993; Anjum et al., 2007), de la proteína de cacahuate (Liu, Tellez \& Castell, 2004; Hwang et al.,2010) y del aislado de la proteína de soya (Gennadios et al., 1993), con respecto a la elongación a la rotura, ésta fue de $124.16 \pm 15.04 \%$ en el presente trabajo y es similar a la de las películas de aislado de la proteína de soya (Gennadios et al., 1993) y de la proteína de cacahuate (Liu et al., 2004; Hwang et al., 2010), tanto la resistencia a la tensión como la elongación a la rotura de películas producidas con mucílago de nopal y glicerol; y de mucílago de nopal, glicerol y calcio en el trabajo de Espino et al. (2010), son menores a las de las películas preparadas con jugo de nopal decantado de $O$. megacantha, proteína animal, glicerol y cera del presente trabajo. El módulo de Young de las películas del presente estudio fue $1.77 \pm 0.42 \mathrm{MPa}$.

\section{Conclusiones}

Se ha determinado la composición química de cuatro variantes del jugo de O. megacantha. El jugo de las cuatro variantes tiene un alto contenido de carbohidratos, siendo el jugo de cladodios con un año de edad el que presenta mayor concentración de ellos, independientemente del manejo que se tenga durante su producción. Además, en todos los jugos estudiados se encontraron compuestos fenólicos que pueden resultar interesantes por su posible actividad antioxidante y su efecto plastificante en películas comestibles. Es factible obtener películas plásticas utilizando jugo decantado de nopal de la especie $O$. megacantha. La combinación de jugo de nopal de $O$. megacantha decantado, proteína animal, glicerol y cera natural da resultados de elongación a la rotura y resistencia a la tensión similares a los de otros biopolímeros utilizados como películas comestibles. Se han identificado tanto carbohidratos como compuestos fenólicos que son de interés en la producción de películas para empaques de alimentos.

\section{Agradecimientos}

Se agradece al Doctorado en Biosistemática, Ecología y Manejo de Recursos Naturales y Agrícolas del Centro Universitario de Ciencias Biológicas y Agropecuarias de la Universidad de Guadalajara (PNPC- CONACYT) y al Centro Universitario de Ciencias Exactas e Ingenierías por el apoyo recibido para la estudiante del Doctorado Sandra Pascoe Ortiz.

\section{CONFLICTO DE INTERESES}

Los autores declaramos que no existe ningún conflicto de intereses.

\section{REFERENCIAS}

Abdel-Hameed, E. S. S., Nagaty, M.A., Salman, M. S. \& Bazaid S. A. (2014). Phytochemicals, nutritionals and antioxidant properties of two prickly pear cactus cultivars (Opuntia ficus-indica Mill.) growing in Taif, KSA. Food Chemistry, 160, 31-38. https://doi.org/10.1016/j. foodchem.2014.03.060.

\section{Ensayo de Tracción}

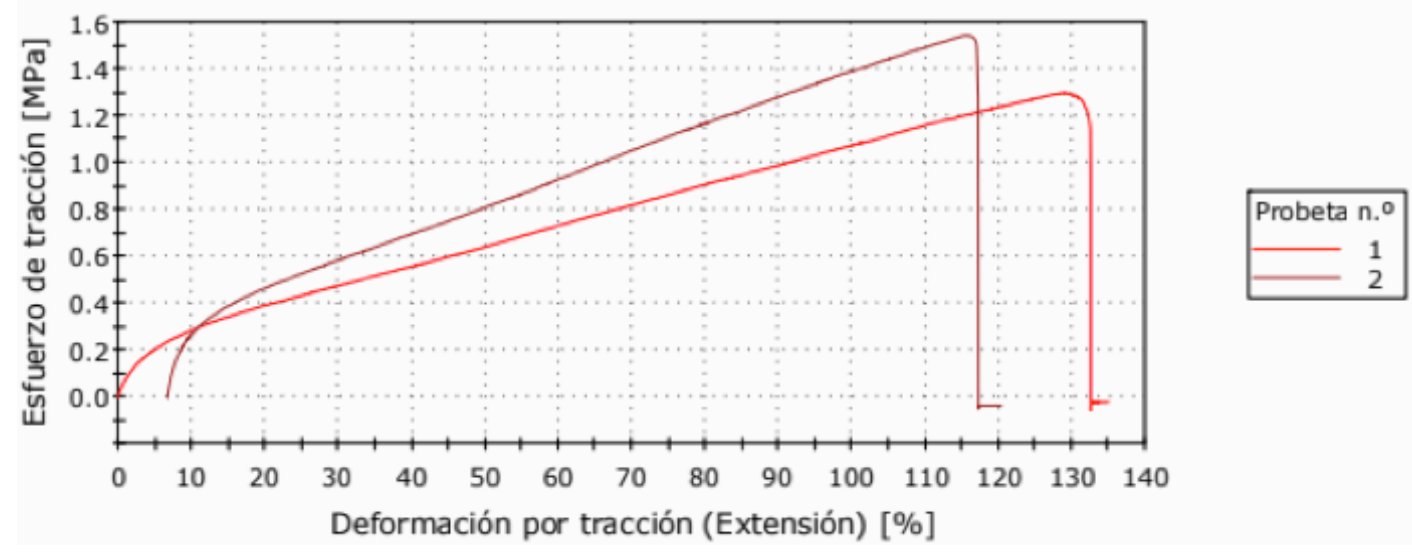

Gráfica 1. Ensayo de tracción en películas de jugo de cladodios silvestres tiernos de O. megacantha. 
Anjum, F. M., Khan, M. R., Din, A., Saeed, M., Pasha, I. \& Arshad, M. U. (2007). Wheat gluten: high molecular weight glutenin subunits-structure, genetics, and relation to dough elasticity. Journal of Food Science, 72(3), R56-63.

AOAC International. (1990). Official methods of analysis. 15th ed. Official Methods: 954.04, 942.05.10, and 962.09.

AOAC International. (1995). Official methods of analysis. 2a ed. Official Method: 930.36 .

AOAC International. (2009). Official methods of analysis. 18th ed. Official Method: 954.02.

Apodaca, P. J., Martínez, M. M., Robles, B. M. \& Rodríguez, F. A. (2016). Polifenoloxidasa, fenoles totales y oscurecimiento de nopal verdura. Revista Mexicana de Ciencias Agrícolas, 7(3), 531-543.

Arcan, I. \& Yemenicioglu, A. (2011). Incorporating phenolic compounds opens a new perspective to use zein films as flexible bioactive packaging materials. Food Research International, 44(2), 550-556. https://doi.org/10.1016/j. foodres.2010.11.034.

Carballo, G. E. T. (2009). Futuro en los Plásticos. Ciencias. Universidad Nacional Autónoma de México. 96, 62-69.

Casas, A., Caballero, J., Valiente, B. A., Soriano, A. \& Dávila, P. (1999). Morphological variation and the process of domestications Stenocereus stellatus (Cactaceae) in Central Mexico. American Journal of Botany, 86(4), 522-533. https://doi.org/10.2307/2656813.

Cenobio, G. A. J., Pimentel, G. D. J., Vargas, T. A., Güemes, V. N. \& Campos, M. R. G. (2015). Efecto de la adición de xoconostle microencapsulado en la actividad antioxidante de películas comestibles de almidón de chayotextle. Boletín de Ciencias Agropecuarias Del ICAP, 1(1). https://doi.org/10.29057/icap.v1i1.977.

Chávez, S. R. A., Gutiérrez, U. J. A. \& Serna, S. S. O. (2009). Phenolic Composition, Antioxidant Capacity and In Vitro Cancer Cell Cytotoxicity of Nine Prickly Pear (Opuntia spp.) Juices. Plant Foods Human Nutrition, 64(2), 146152. https://doi.org/10.1007/s11130-009-0117-0.

Ciannamea, E. M., Stefani, P. M. \& Ruseckaite, R. A. (2016). Properties and antioxidant activity of soy protein concentrate films incorporated with red grape extract processed by casting and compression molding. LWTFood Science and Technology, 74(C), 353-362. https:// doi.org/10.1016/j.lwt.2016.07.073.

Contreras, P. M., Gutiérrez, C. E., Valderrama, B. M. del C., Rojas, M. I., Espinosa, A. D. G. Suárez, V. R. \& Rodríguez, G. M. E. (2012). Effects of Drying Process on the physicochemical Properties of Nopal Cladodes at Different Maturity. Plant Foods Human Nutrition, 67(1), 44-49. https://doi.org/10.1007/s11130-011-0265-X.

Coria, C. Y. S., Ochoa, M. J. \& Nazareno, M. A. (2011). Health-promoting substances and antioxidant properties of Opuntia sp. fruits. Changes in bioactivecompound contents during ripening process. Food
Chemistry, 126, 514-519. https://doi.org/10.1016/j. foodchem.2010.11.033.

Domínguez, C. M. F. \& Jiménez, M. M. T. (2012). Películas comestibles formuladas con polisacáridos: propiedades y aplicaciones. Temas Selectos de Ingeniería de Alimentos, 6(2), 110-121.

Espino, D. M., Ornelas, P. J. De J., Martínez, T. M. A., Santillán, C., Barbosa, C. G. V., Zamudio, F. P. B. \& Olivas, G. I. (2010). Development and characterization of edible films based on mucilage of Opuntia ficus-indica (L.). Journal of Food Science, 75(6), E347-E352. https:// doi.org/10.1111/j.1750-3841.2010.01661.x.

Fabra, M. J., Talens, P. \& Chiralt, A. (2010). Influence of calcium on tensile, optical and water vapour permeability properties of sodium caseinate edible films. Journal of Food Engineering, 96(3), 356-364. https://doi. org/10.1016/j.jfoodeng.2009.08.010.

Fernández, L. J. A., Almeda, L., Obón, J. M. \& Castellar, R. (2010). Determination of antioxidant constituents in cactus pear fruits. Plant food for human nutrition, 65, 253-259. https://doi.org/10.1007/s11130-010-0189-x.

Figueroa, C. I., Martínez, D. M. T., Rodríguez, P. E., Colinas, L. M. T., Valle, G. S., Ramírez, R. S. \& Gallegos, V. C. (2010). Pigments content, other compounds and antioxidant capacity in 12 cactus pear cultivars (Opuntia spp.) from México. Agrociencia, 44(7), 763-771.

Flores, A. M. C., Vergara, B. F. T. \& Guerrero, B. J. A. (2011). Efecto del tiempo de almacenamiento y tipo de procesamiento en los antioxidantes de nopal. Temas selectos de ingeniería de alimentos, 5 (2), 84-96.

Gallegos, V. C., Scheinvar, L., Núñez, C. C. A. \& Mondragón, J. C. (2012). Morphological diversity of xoconostles (Opuntia spp.) or acidic cactus pears: a Mexican contribution to functional foods. Fruits, 67,109-120. https://doi.org/10.1051/fruits/2012001.

García, N. F., Peña, V. C. B., Trejo, C., García, N. R., Reyes, A. J. A. \& Aguirre, R. J. R. (2015). Biophysical and physiological characteristics of nopalitos (Opuntia spp., Cactaceae) as influenced by domestication. Genetic Resources and Crop Evolution, 62(6), 927-938. https:// doi.org/10.1007/s10722-014-0201-7.

Gennadios, A., Brandenburg, A.H., Weller, C.L. \& Testin, R.F. (1993). Effect of $\mathrm{pH}$ on properties of wheat gluten and soy protein isolate films. Journal of Agricultural and Food Chemistry, 41, 1835-9.

González, D. A., Riojas, L. M. E. \& Arreola, N. H. J. (2001). El género Opuntia en Jalisco. Guía de campo. Universidad de Guadalajara. México, 62.

González, M. G., Bernal, R. O., Gutiérrez, A. E. E. \& Santamaría, M. E. A. (2018). Usos y aplicaciones del nopal como oportunidad competitiva en la Ciudad de Toluca, Vinculatégica EFAN, 3(2), 73-79.

Guevara, T., Jiménez, H., Reyes, M. L., Mortensen, A. G., Laursen, B. B., Lin, L. W., De Léon, R. A., Fomsgaard, I. S. 
\& Barba de la Rosa, A. P. (2010). Proximate composition, phenolicacids, and flavonoids characterization of commercial and wild nopal (Opuntia spp.). Journal of Food Composition and Analysis, 23(6), 525-532. https:// doi.org/10.1016/j.jfca.2009.12.003.

Guzmán, L. D., \& Chávez, J. (2007). Estudio bromatológico del cladodio del nopal (Opuntia ficus-indica) para el consumo humano. Revista de la Sociedad Química del Perú, 73(1), 41-45.

Harborne, J. B. (1998). Phytochemical Methods; A Guide to Modern Techniques of Plant Analysis, third ed. Chapman and Hall, New York.

Hwang, J. Y., Shyu, Y. S., Wang, Y. T. \& Hsu, C. K. (2010). Antioxidative properties of protein hydrolysate from defatted peanut kernels treated with esperase. LWT Food Science and Technology, 43(2), 285-90.

Jiménez, A. D. M., López, M. J. M., Hernández, B. C., Gutiérrez, U. J. A. \& Welti, C. J. (2015). Dietary fiber, phytochemical composition and antioxidant activity of Mexican commercial varieties of cactus pear. Journal of Food Composition and Analysis, 41, 66-73. https://doi. org/10.1016/j.jfca.2015.01.017.

Knishinsky, R. (2004). Prickly pear cactus medicine: Treatments for diabetes, cholesterol, and the immune system. United States: Inner Traditions/Bear, 20-86.

Liu, C. C., Tellez, G. A. M. \& Castell, P. M. E. (2004). Physical and mechanical properties of peanut protein films. $L W T-$ Food Science and Technology, 37, 731-738.

López, P. C. \& Peña, V. C. B. (2016). Variación en el contenido de algunos metabolitos secundarios nopalitos de diferentes especies (Opuntia spp.). Proceedings of the international agroindustrial Engineering Conference. Mesa 2. Alimentos funcionales e innovadores. 1, 4.

López, P. C., Peña, V. C. B., Rodríguez, H. A. I. \& Reyes, A. J. A. (2016). Rheological flow behavior of structural polysaccharides from edible tender cladodes of wild, semidomesticated and cultivated 'nopal' (Opuntia) of mexican highlands. Plant Foods for Human Nutrition, 71(4), 388-395. https://doi.org/10.1007/s11130-0160573-2.

Loveys, B. R., Stoll, M. \& Davies, W. J. (2004). Physiological approaches to enhance water use efficiency in agriculture: exploiting plant signaling in novel irrigation practice. In: Bacon M. A. Water use efficiency in plant biology. Blackwell Publishing Ltd, London, 113-141.

McGarvie, D. \& Parolis, H. (1979). The mucilage of Opuntia ficus-indica. Carbohydrate Research, 69, 171-179. https://doi.org/10.1016/S0008-6215(00)85762-6.

Mondragón, J. C. \& Pérez, G. S. (2003). El nopal (Opuntia spp.) como forraje. Estudio FAO Producción y Protección Vegetal. Roma. 169, 98.

Moussa-Ayoub, T. E., El-Samahy, S. S., Rohn, S. \& Kroh, L. W. (2011). Flavonols, betacyanins content and antioxidant activity of cactus Opuntia macrorrhiza fruits.
Food Research International, 44, 2169-2174. https://doi. org/10.1016/j.foodres.2011.02.014.

Ndhlala, A. R., Kasiyamhuru, A., Mupure, C., Chitindingu, K., Benhura, M. A. \& Muchuweti, M. (2007). Phenolic composition of Flacourtia indica, Opuntia megacantha and Sclerocary abirrea. Food Chemistry, 103, 82-87. https://doi.org/10.1016/j.foodchem.2006.06.066.

Nie, X., Gong, Y., Wang, N. \& Meng, X. (2015). Preparation and characterization of edible myofibrillar proteinbased film incorporated with grape seed procyanidins and green tea polyphenol. LWT - Food Science and Technology, 64, 1042-1046. https://doi.org/10.1016/j. lwt.2015.07.006.

Nobel, P. S., Cavelier, J. \& Andrade, J. L. (1992). Mucilage in cacti: its apoplastic capacitance, associated solutes, and influence on tissue 5. Journal of Experimental Botany, 43, 641-648. https://doi.org/10.1093/jxb/43.5.641.

Osorio, E. O., Ortiz, M. A., Álvarez, V. B., Dorantes, A. L. \& Giusti, M. M. (2011). Phenolics, betacyanins and antioxidant activity in Opuntia joconostle fruits. Food Research International, 44, 2160-2168. https://doi. org/10.1016/j.foodres.2011.02.011.

Pan, Y., Wang, K., Huang, S., Wang, H., Mu, X. \& He, C. (2008). Antioxidant activity of microwave-assisted extraction of logan (Dimocarpus longan Lour.) peel. Food Hydrocolloids, 32, 35-41. https://doi.org/10.1016/j. foodchem.2007.07.033.

Paulsen, B. S. \& Lund, P. S. (1979). Water-soluble polysaccharides of Opuntia ficus-indica cv "Burbank's Spineless". Phytochemistry, 18, 569-571. https://doi. org/10.1016/S0031-9422(00)84262-4.

Pimienta, E. (1990). El nopal tunero. Universidad de Guadalajara. México.

Ramírez, T. H. M., Reyes, A. J. A., Pinos, R. J. M. \& Aguirre, R. J. R. (2007). Efecto de la especie y madurez sobre el contenido de nutrientes de cladodios de nopal. Agrociencia, 41(6), 619-626.

Reid, G. J. S. (1985). Cell wall storage carbohydrates in seeds: biochemistry of seed "gums" and "hemicelluloses". Advances in Botanical Research, 11, 125-155. https:// doi.org/10.1016/S0065-2296(08)60170-6.

Repo, de C. R. \& Encina, Z. C. R. (2008). Determinación de la capacidad antioxidante y compuestos bioactivos de frutas nativas peruanas. Revista de la Sociedad Química del Perú, 74(2), 108-124.

Restrepo, F. J. I. \& Aristizábal, T. I. D. (2010). Conservation of strawberry (Fragaria x ananassa Duch cv. Camarosa) by edible coating application of sabila gel mucilage (Aloe barbadensis Miller) and carnauba wax. Revista de la Facultad de Química Farmacéutica, Universidad de Antioquía, 17(3), 252-263.

Ribeiro, E. M. Silva, N. H., Filho, J. L., Brito, J. Z. \& Silva, M. D. (2010). Study of carbohydrates present in the cladodes of Opuntia ficus-indica (fodder palm), 
according to age and season. Ciência e Tecnología de Alimentos, 30(4), 933-939. https://doi.org/10.1590/ S0101-20612010000400015.

Rodríguez, G. M. E., De Lira C., Hernández B. E., Cornejo, V. M. A., Palacios F. A. J., Rojas, M. I., Reynoso, R., Quintero, L. C., Del Real, A., Zepeda, T. A. \& Muñoz, T. C. (2007). Physicochemical characterization of nopal pads (Opuntia ficus-indica) and dry vacuum nopal powders as a function of the maturation. Plant Foods for Human Nutrition, 62(3), 107-112. https://doi.org/10.1007/ s11130-007-0049-5.

Ruiz, A. G. (2006). Obtención y caracterización de un polímero biodegradable a partir del almidón de yuca. Ingeniería y Ciencia, 2(4), 5-28.

Saberi, B., Chockchaisawasdee, S., Golding, J. B., Scarlett, C. J. \& Stathpoulos, C. E. (2017). Physical and mechanical properties of a new edible film made of pea starch and guar gum as affected by glycols, sugars and polyols. International Journal of Biological Macromolecules, 104(Pt A), 345-359. https://doi.org/10.1016/j.ijbiomac. 2017.06 .051 .

Sáenz, C. (1997). Cactus cladodes: a source of dietary fiber. Journal of the Professional Association for Cactus Development, 2, 117-123.

Sáenz, C. (2006).Utilización agroindustrial del nopal. Food \& Agriculture Org., 162, 2-4.

Saura, C. F. (2010). Dietary Fiber as a Carrier of Dietary Antioxidants: An Essential Physiological Function. Journal of Agricultural and Food Chemistry, 59(1), 4349. https://doi.org/10.1021 / jf1036596.

SAGARPA. Secretaría de Agricultura, Ganadería, Desarrollo Rural, Pesca y Alimentación. (2015). Estudio de factibilidad para el establecimiento de cultivo de nopal (Opuntia) en tierras ociosas en los estados de Aguascalientes, San Luis Potosí, Guanajuato y Zacatecas con fines alimenticios, energéticos y ambientales, 39.

SAGARPA. Secretaría de Agricultura, Ganadería, Desarrollo Rural, Pesca y Alimentación. (2016). Cierre de la producción agrícola por cultivo. Servicio de Información Agroalimentaria y Pesquera.

Sumaya, M. M. T., Cruz, J. S., Madrigal, S. E., García, P. J. D., Cariño, C. R., Cruz, C. N., Valadez, V. C., Martínez, C. L \& Alanís, G. E. (2011). Betalain, acid ascorbic, phenolic contents and antioxidant properties of purple, red, yellow and white cactus pears. International Journal of Molecular Sciences, 12, 6452-6468. https://doi. org/10.3390/ijms12106452.

Tharanathan, R. (2003). Biodegradable films and composite coatings: past, present and future. Critical Review in Foods Science and Technology, 14, 71-78. https://doi. org/10.1016/S0924-2244(02)00280-7.

Trachtenberg, S. \& Mayer, A. M. (1981). Composition and properties of Opuntia ficus-indica mucilage. Phytochemistry, 20, 2665-2668. https://doi. org/10.1016/0031-9422(81)85263-6.

Torres, P. R. L., Morales, C. D., Ballinas, C. M. de L. \& Nevárez M. G. V. (2015). El nopal: planta del semidesierto con aplicaciones en farmacia, alimentos y nutrición animal. Revista Mexicana de Ciencias Agrícolas, 6 (5), 1129-1142.

Torres, S. A. (2010). Sistemas de producción de nopal forrajero en Brasil. Revista Salud Pública y Nutrición, Edición Especial. 5, 57-69.

Valencia, S. K., Brambila, P. J. de J. \& Mora, F. J. S. (2010). Nopal evaluation as functional food by means of real option. Agrociencia, 44 (8), 955-963.

Valero, V. M. F., Ortegón, Y., \& Uscategui, Y. (2013). Biopolímeros: Avances y Perspectivas. Dyna, 80(181), 171-180.

Wang, L., Dong Y., Men, H., Tong, J. \& Zhou, J. (2008). Preparation and characterization of active films based on chitosan incorporated tea polyphenols. Food Hydrocolloids, 32, 35-41. https://doi.org/10.1016/j. foodhyd.2012.11.034.

Waterman, P. G. \& Mole, S. (1994). Analysis of phenolic plant metabolites. Oxford: Blackwell Scientific Publications, 105-133.

Yeddes, N., Chérif, J. K., Guyot, S., Sotin, H. \& Ayadi, M. T. (2013). Comparative study of antioxidant power, polyphenols, flavonoids and betacyanins of the peel and pulp of three Tunisian Opuntia forms. Antioxidants, 2(2), 37-51. https://doi.org/10.3390/antiox2020037.

Zamudio, F. P. B., Bello, P. L. A., Vargas, T. A., Hernández, U. J. P. \& Romero, B. C. A. (2007). Caracterización parcial de películas preparadas con almidón oxidado de plátano. Agrociencia, 41, 837-844. 